\title{
Percepción del clima organizacional en la escuela y su incidencia en la educación
}

\author{
Perception of the organizational climate at school and its impact on education \\ Percepção do clima organizacional na escola e seu impacto na educação
}

Nain Maraza Vilcanqui ${ }^{1}$

marazanain@unap.edu.pe

https://orcid.org/0000-0002-9313-2947

\author{
Gladys Martha Flores Choque ${ }^{3}$ \\ gladysmarthaf@gmail.com \\ https://orcid.org/0000-0002-9004-6170
}

\author{
Beker Maraza Vilcanqui ${ }^{2}$ \\ bmarazav@unia.edu.pe \\ https://orcid.org/0000-0002-0155-4094
}

\author{
Queke Maraza Vilcanqui ${ }^{3}$ \\ milqueades@gmail.com \\ https://orcid.org/0000-0002-5505-0844
}

${ }^{1}$ Universidad Nacional del Altiplano, Puno-Perú

${ }^{2}$ Universidad Nacional Intercultural de la Amazonía, Pucallpa-Perú ${ }^{3}$ Instituto de Investigación de las Culturas Andinas y Amazónicas, Puno-Perú

Artículo recibido 23 de noviembre 2021, arbitrado y aceptado 15 de diciembre 2021 y publicado 7 de marzo 2022

\section{ABSTRACT}

La investigación se realizó con el objetivo de identificar la Percepción del Clima Organizacional en docentes y administrativos de la Institución Educativa Primaria N 70081 de Salcedo Puno 2017. El tipo de estudio fue descriptivo; la muestra estuvo conformado por 15 unidades de estudio entre docentes y administrativo; para la recolección de datos se aplicó el cuestionario CLIOUNing, que mide las percepciones sobre el clima organizacional de acuerdo a las dimensiones que consiente dicho instrumento (Gestión Institucional, Retos personales e Interacción Institucional); con siguientes resultados: del promedio total, nos permiten apreciar que el $53.9 \%$ de satisfacción, donde se evidencia una percepción medianamente favorable del clima organizacional; el segundo conglomerado representa 30.3 $\%$ el cual indica un clima favorable y el menor conglomerado, indica $15.7 \%$ que representa un clima organizacional desfavorable, se concluye que existe un clima organizacional mediamente favorable.

Palabras clave: Clima Organizacional; Gestión Institucional; Interacción Institucional; Retos Personales

\section{RESUMEN}

The research was conducted with the objective of identifying the Perception of the Organizational Climate in teachers and administrative staff of the Primary Educational Institution $\mathrm{N}^{\circ} 70081$ of Salcedo Puno 2017. The type of study was descriptive; the sample consisted of 15 study units between teachers and administrative; for data collection the CLIOUNing questionnaire was applied, which measures perceptions about the organizational climate according to the dimensions that this instrument consents (Institutional Management, Personal Challenges and Institutional Interaction); with the following results: of the total average, allow us to appreciate that $53.9 \%$ of satisfaction, where a moderately favorable perception of the organizational climate is evidenced; the second conglomerate represents 30.3 $\%$ which indicates a favorable climate and the smallest conglomerate indicates $15.7 \%$ which represents an unfavorable organizational climate, it is concluded that there is a moderately favorable organizational climate.

Key words: Organizational Climate; Institutional Management; Institutional Interaction; Personal Challenges

\section{RESUMO}

A pesquisa foi conduzida com o objetivo de identificar a Percepção do Clima Organizacional em professores e pessoal administrativo da Instituição de Ensino Primário N 70081 de Salcedo Puno 2017. O tipo de estudo foi descritivo; a amostra consistiu de 15 unidades de estudoincluindo professores e pessoal administrativo; para a coleta de dados, foi aplicado o questionário CLIOUNing, que mede percepções do clima organizacional de acordo com as dimensões do instrumento (Gestão Institucional, Desafios Pessoais e Interação Institucional); com os seguintes resultados: da média total, podemos apreciar que 53. $9 \%$ de satisfação, onde uma percepção moderadamente favorável do clima organizacional é evidente; o segundo grupo representa $30,3 \%$ que indica um clima favorável e o menor grupo indica $15,7 \%$ que representa um clima organizacional desfavorável, conclui-se que existe um clima organizacional moderadamente favorável.

Palavras-chave: Clima Organizacional; Gestão Institucional; Interação Institucional; Desafios Pessoais 


\section{INTRODUCCIÓN}

El clima organizacional puede considerarse como un medio para el éxito de una determinada organización, muchas organizaciones han demostrado que este tema representa un método que permite conocer el curso de su organización en relación a ciertos criterios importantes. Los orígenes teóricos de esta definición no están siempre claros en el desarrollo de muchas organizaciones. Además, nace las explicaciones a raíz de acciones del hombre integrado en una organización, ante esta situación las relaciones son susceptibles de crear o suscitar escenarios de discrepancia, confrontación, ocasionada por diversos factores como mala comunicación, la ausencia del diálogo y escucha activa, liderazgo, entre muchos otros (Esquivel et al., 2020).

Por consiguiente, el Clima Organizacional es la descripción individual del marco social o contextual del cual forma parte una persona; son percepciones compartidas de políticas, prácticas y procedimientos organizaciones tanto formales como informales.(Chiang, et al., 2017).

Un estudio sobre la satisfacción laboral dentro del clima organización permite generar una evaluación sobre la situación laboral de los funcionarios, su forma de su organización y que ello ayudaría en la implementación de estrategias dirigidas a mejorar el entorno laboral, considerando que el recurso humano cumple un rol fundamental en el logro de metas de una institución. (Montoya et al., 2017).
El desarrollo de las competencias socio afectivas favorece la adquisición de valores y conductas relacionados con el trabajo cooperativo (Cortés y Rosales, 2019), si bien es cierto que la aplicación de una estrategia en el interior de una organización favorece grandemente en evitar conflictos y desempeñarse correctamente enlos trabajadores.Por consiguiente, los resultados de un estudio mostraron la relación bilateral positiva y estadísticamente significativa entre el clima organizacional y la satisfacción laboral, significa que existe una relación entre el clima organizacional favorable con el grado de satisfacción laboral en trabajadores de una organización. Pupo et al., (2017) expresa que un clima organizacional adecuado beneficia la salud psicológica de los trabajadores y a su vez un clima organizacional modificado genera implicaciones psicológicas en los trabajadores. Para Chiang, Heredia, et al., (2017) el clima organizacional es muy importante en la gestión de las organizaciones es estudiado para obtener un alto desempeño laboral, eleva el compromiso y la cooperación entre los participantes del grupo de trabajo de manera satisfactorio. Zambrano et al., (2017) en tanto, dependerá del tipo de líder que influirá en los trabajadores, interviniendo en el actuar de estos de tal forma que genera una motivación que permite el máximo desempeño en busca de la excelencia organizacional. Para Salguero y García, (2017) si se tiene o se buscan altos niveles de liderazgo, podrían traducirse en mejoras del clima organizacional. Castillo et al., (2019), asimismo plantea que el rol 
del líder incide positivamente sobre las conductas de apoyo y de orientación a las reglas y sobre la autonomía y profesionalismo, ambas dimensiones se potencien mutuamente, de forma tal que el clima mejore influyendo en la mejor disposición de los trabajadores. Adrianzola et al., (2019) entonces, se puede afirmar que las instituciones tienen un clima organizacional agradable, debido a que permite a cada uno de los empleados desarrollar sus funciones de manera óptima. Cota, (2017) por consiguiente, el factor humano la más importante en una organización, a quien se debe prestar la debida atención para mantener elevado su nivel emocional y de satisfacción, impidiendo que llegue a realizar actividades que se vuelvan monótonas y sin sentido. Meza, (2017),expresa que los estudios realizados en docentes, ciertos resultados están satisfechos con el clima organizacional, indistintamente del sistema de valores. Riveros y Grimaldo (2017) menciona que estos hallazgos permiten cada vez mejorar en las organizaciones su satisfacción con y en grupos de trabajo.

Para tener éxito en las organizaciones la motivación va acompañado en las personas, tal como el estudio indica que la realidad económica, es un condicionante influyente en el factor de motivación laboral (Pazmay y Ortíz, 2018). Asimismo, la felicidad como un foco generador de satisfacción laboral, compromiso organizacional, capacidad innovadora, solución de problemas, mejora de relaciones interpersonales y productividad (Diaz y Carrasco, 2018), por ende, el clima organizacional es fundamental para las organizaciones de modo que estas puedan lograr sus objetivos y que de manera más específica es importante que estas mismas entiendan los factores que conforman el clima organizacional (Parra et al., 2018), mientras tanto, la calidad de vida laboral de una organización debe ser el entorno de trabajo, el ambiente favorable en la organización que permita mejorar la calidad en la ejecución de las actividades laborales, mejorar el clima organizacional y la contribución óptima a la empresa por parte del trabajador que posee confianza y respeto (Cardona et al., 2018). Como la calidad de vida laboral de los trabajadores los cuales son factor clave para la productividad de una organización. (Suárez, 2018), no se pueden distinguir la participación del género, porque todos ellos contribuyen satisfactoriamente, tanto hombres y mujeres, hay una marcada participación de los hombres en estas entidades, sin embargo, las mujeres son las que mayores niveles de formación presentan (González et al., 2018). Según la investigación se tiene la existencia de un clima desfavorable de acuerdo a la percepción de los propios trabajadores en el sector público (Charry, 2018); como también el clima organizacional en el ambiente laboral de la Empresas Sociales de Estado en general es adecuado (positivo) (Rivera et al., 2018). Frente a las entidades públicas o privadas, se tienen resultados que quienes trabajan en el sector privado califican más alto este factor que los empleados del sector público en la identidad y la oportunidad de crecer en un clima organizacional favorable y aportar el crecimiento de la organización (Pedraza y Bernal, 2018), el crecimiento del clima organizacional 
dependerá de manejar adecuadamente los variables como el liderazgo, el compromiso organizacional, el desempeño laboral, la motivación, el trabajo en equipo, la cultura organizacional, representan algunas de las líneas de investigación futuras en las organizaciones públicas y privadas (Pedraza, 2018).

Un aspecto fundamental en el crecimiento del ser humano es la actitud y el comportamiento dentro de una organización, que las actitudes laborales influyen en forma directa y como mediadora del clima organizacional sobre el comportamiento prosocial, ratificando el hecho que las actitudes laborales transfieren indirectamente parte del efecto del clima organizacional sobre los factores de comportamiento prosocial (Lapo y Bustamante, 2018). Se puede expresar en las relaciones interpersonales entre compañeros $y$ con los superiores representa ser el componente del clima organizacional que mayor impacto produce sobre la satisfacción del personal hacia su centro de trabajo (Soria et al., 2019), un aspecto a considerar para las investigaciones futuras sobre la inteligencia interpersonal y su incidencia en clima organizacional favorable y las propuestas acerca de la constante mejora y cultivar en los colaboradores generen confianza, empoderamiento y compromiso de los mismo para afrontar los retos presentes y futuros (Brito et al., 2020).

La economía informacional y las Tecnologías de Información y Comunicación, resultan estratégicos para el desarrollo organizacional en la medida que contribuyen con el proceso de innovación y desarrollo tecnológico, dirigido hacia el mejoramiento de algunos aspectos clave de la organización que permiten incrementar su competitividad en los mercados globalizados
(Panchi, 2018). Por otra parte, el usuario es el mejor testigo para calificar la calidad de atención y ser satisfechos dentro de una organización humana, tal cual ocurre en los establecimientos de salud y educación dónde el cliente tiene la razón, lo propio debe evidenciar la dimensión técnica científica aguardando coherencia y correspondencia con la dimensión humana para lograr que se valore la calidad de la atención y eficiencia (Fernández et al., 2019).

La constante mejora dependerá de la evaluación permanente del personal humano en las organizaciones, dado que se deben fijar mecanismos o procedimientos que permitan la continua y sistemática evaluación del clima organizacional para tener puntos de referencia en cuanto a la mejora y los avances (Brito, 2018), Los resultados de las investigaciones contribuyen a las mejoras de las organizaciones, quienes deben enfocar sus esfuerzos en monitorear y mejorar constantemente cada uno de los aspectos del clima organizacional analizados, sin duda, influyen de forma importante en la satisfacción de cada uno de sus empleados, esto permitiría en una mayor productividad y mejor servicio a los pacientes en el campo de la salud (Ortiz et al., 2019), cuando las personas trabajan en una organización y se sienten contentas con la función desempeñada, el compromiso con esta aumenta y mejoran los resultados (Chirinos et al., 2018), por consiguiente es concordante con el estudio de la percepción del clima organizacional de docentes de educación para personas adultas, la gestión directiva juega un rol protagónico en la motivación y la generación de compromiso (Sagredo y Castelló, 2019). 
El campo de la educación y formación humana es importante la gestión de recursos humanos por lo que el estudio realizado señala la importancia de la gestión que los directores y sus equipos directivos deben asumir para mejorar positivamente en procesos formativos de trabajo en equipo con optimismo y flexibilidad para encarar los retos y las metas establecidas (López et al., 2019). Para lo cual se pueden emplear estrategias de gestión de recursos humanos apropiadas y concretas para motivar y retener a la gente y mejorar su desempeño laboral (Luna et al., 2019).

En relación al sector de la educación Niebles et al., (2019) plantea que:

En el campo pedagógico se tiene que el clima organizacional es muy importante tal cual indican que en universidades privadas de Barranquilla Colombia, cuentan con un ambiente en el que factores como el liderazgo, el trabajo cooperativo la motivación, las relaciones interpersonales y la toma de decisiones, gestan un clima organizacional positivo, donde los objetivos organizacionales se logran en forma efectiva, a la luz del trabajo conjunto (Niebles et al., 2019).

La toma de decisiones que promueve la mejora en los procesos y resultados de la investigación, sin duda, contribuye en la mejora continua en el desarrollo humano y en las prevenciones sobre las conductas no adecuadas en los trabajadores en la Educación Básica Regular o en distintos ámbitos de la gestión pública (Hirsch, 2016). Entre ellos, existen otros factores para su mejora, así como factores que inciden directamente al personal, entonces se recomienda a las instituciones educativas a promover un adecuado clima institucional, con principios de sostenibilidad e inclusivo en el futuro, además estas mejoras contribuyan a disminuir el estrés en el trabajo diario, de mantenerse se pueden generar problemas patológicos que afectarían la salud mental y la calidad de vida en docentes (Bada et al., 2020).

\section{MÉTODO}

La investigación fue cuantitativa con diseño descriptivo con una población de 15 docentes, como muestra se seleccionó el total de la población clasificada en 13 docentes y 2 administrativos de la Institución Educativa Primaria de salcedo Puno. Para la recogida de los datos se aplicó la técnica de encuesta y como instrumento el cuestionario compuesto por una escala de actitudes aprobado por M.Sc. Agustín Alexander Mejías Acosta, Oscar Arzola Minerva, los cuales se aplicaron para recoger la información de las dimensiones de la gestión institucional, clima organizacional e interacción institucional.

\section{RESULTADOS Y DISCUSIÓN}

\section{Clima organizacional de docentes y administrativos que laboran en la Institución Educativa Primaria N $^{\circ} 70071$ de Salcedo Puno}

Los resultados encontrados en la investigación acerca de la percepción del clima organizacional en docentes y administrativos en las tres dimensiones, así como muestra en la Tabla 1. 
Tabla 1. Resultados de la dimensión gestión institucional.

\begin{tabular}{|c|c|c|c|c|c|c|c|c|c|}
\hline \multirow[t]{2}{*}{ Dimensión } & \multirow{2}{*}{$\begin{array}{l}\text { Categorías } \\
\text { Indicador }\end{array}$} & \multicolumn{2}{|c|}{ Desfavorable } & \multicolumn{2}{|c|}{$\begin{array}{l}\text { Medianamente } \\
\text { favorable }\end{array}$} & \multicolumn{2}{|c|}{ Favorable } & \multicolumn{2}{|c|}{ TOTAL } \\
\hline & & Fcia & $\%$ & Fcia & $\%$ & Fcia & $\%$ & Fcia & $\%$ \\
\hline \multirow[t]{4}{*}{$\begin{array}{l}\text { Gestión } \\
\text { institucional }\end{array}$} & $\begin{array}{l}\text { 1. Su institución es reconocida por su } \\
\text { gestión de calidad. }\end{array}$ & 2 & 13.3 & 1 & 6.7 & 12 & 80,0 & 15 & 100.0 \\
\hline & $\begin{array}{l}\text { 2. Tiene una planificación institucional } \\
\text { que guía sus actividades. }\end{array}$ & 2 & 13.3 & 11 & 73.3 & 2 & 13.3 & 15 & 100.0 \\
\hline & $\begin{array}{l}\text { 3. Su trabajo está delimitado y } \\
\text { estructurado. }\end{array}$ & 1 & 6.7 & 12 & 80.0 & 2 & 13.3 & 15 & 100.0 \\
\hline & 4. Pertenece a un buen equipo. & 2 & 13.3 & 5 & 33.3 & 8 & 53.3 & 15 & 100.0 \\
\hline TOTAL & & 7 & 11.6 & 29 & 48.3 & 24 & 40.0 & 60 & 100.0 \\
\hline
\end{tabular}

Cuestionario CLIOUNing aplicado por el investigador al personal docente y administrativo.

Los resultados presentados del promedio total presente en la Tabla 1 permiten apreciar que el mayor puntaje obtenido es 29 puntos que equivale al $48.3 \%$ de satisfacción, del total de la población analizada donde se evidencia una percepción medianamente favorable del gestión institucional; el segundo conglomerado nos permite considerar al puntaje obtenido de 24 puntos que equivale al 40 $\%$, del total de la población analizada el cual indica un clima favorable de gestión institucional; y el menor conglomerado, es igual al puntaje obtenido de 7 puntos que equivale al $11.6 \%$, del total de la población analizada el cual nos indica un clima desfavorable del gestión institucional.

Como es evidente y a la luz de los resultados observados el $48.3 \%$ de la población se muestra satisfecha respecto a la gestión institucional, el clima organizacional del personal que labora en la Institución Educativa Primaria de Salcedo, responde medianamente favorable ante los lineamientos establecidos dentro de la institución, aspectos formales como reglas, normas, manuales de función y organizaciones, la planificación establecida dentro de la organización, la delimitación de funciones y responsabilidades. Así mismo tiene un sentido de pertenencia e identificación con un equipo de trabajo bueno.

Los resultados presentados del promedio total en la Tabla 2 permiten apreciar que el mayor puntaje obtenido es 31 puntos que equivale al $68.8 \%$ de satisfacción, del total de la población analizada donde se evidencia una percepción medianamente favorable de retos personales; el segundo conglomerado nos permite considerar al puntaje obtenido de 9 puntos que equivale al $20 \%$, del total de la población analizada el cual indica un clima favorable de retos personales; y el menor conglomerado, es igual al puntaje obtenido de 5 puntos que equivale al $11.1 \%$, del total de la población analizada el cual nos indica un clima desfavorable de retos personales. 
Tabla 2. Resultados de la dimensión retos personales.

\begin{tabular}{|c|c|c|c|c|c|c|c|c|c|}
\hline \multirow[t]{2}{*}{ Dimensión } & \multirow{2}{*}{$\begin{array}{l}\text { Categorías } \\
\text { Indicador }\end{array}$} & \multicolumn{2}{|c|}{ Desfavorable } & \multicolumn{2}{|c|}{$\begin{array}{l}\text { Medianamente } \\
\text { favorable }\end{array}$} & \multicolumn{2}{|c|}{ Favorable } & \multicolumn{2}{|c|}{ TOTAL } \\
\hline & & Fcia & $\%$ & Fcia & $\%$ & Fcia & $\%$ & Fcia & $\%$ \\
\hline \multirow[t]{3}{*}{$\begin{array}{l}\text { Retos } \\
\text { personales }\end{array}$} & $\begin{array}{l}\text { 1. Se adapta rápidamente a nuevas } \\
\text { situaciones. }\end{array}$ & 2 & 13.3 & 9 & 60.0 & 4 & 26,7 & 15 & 100.0 \\
\hline & $\begin{array}{l}\text { 2. Desarrolla sus habilidades y } \\
\text { conocimientos. }\end{array}$ & 1 & 6.7 & 11 & 73.3 & 3 & 20.0 & 15 & 100.0 \\
\hline & $\begin{array}{l}\text { 3. Mantiene altos estándares de } \\
\text { desempeño.. }\end{array}$ & 2 & 13.3 & 11 & 73.3 & 2 & 13.3 & 15 & 100.0 \\
\hline TOTAL & & 5 & 11.1 & 31 & 68.8 & 9 & 20.0 & 45 & 100.0 \\
\hline
\end{tabular}

Cuestionario CLIOUNing aplicado por el investigador al personal docente y administrativo.

Como es evidente y a la luz de los resultados observados el $68.8 \%$ de la población se muestra satisfecha respecto a retos personales, el clima organizacional del personal que labora en la Institución Educativa Primaria de Salcedo, responde medianamente favorable ante los lineamientos establecidos dentro de la institución, aspectos formales como reglas, normas, manuales de función y organizaciones, la planificación establecida dentro de la organización, la delimitación de funciones y responsabilidades. Así mismo tiene un sentido de pertenencia e identificación con un equipo de trabajo bueno.

Los resultados del promedio total en la Tabla 3 permiten apreciar que el mayor puntaje obtenido es 29 puntos que equivale al $48.3 \%$ de satisfacción, del total de la población analizada donde se evidencia una percepción medianamente favorable de interacción institucional; el segundo conglomerado nos permite considerar al puntaje obtenido de 17 puntos que equivale al $28.3 \%$, del total de la población analizada el cual indica un clima favorable de interacción institucional; y el menor conglomerado, es igual al puntaje obtenido de 14 puntos que equivale al $23.3 \%$, del total de la población analizada el cual nos indica un clima desfavorable de interacción institucional. 
Tabla 3. Resultados de la dimensión interacción institucional.

\begin{tabular}{|c|c|c|c|c|c|c|c|c|c|}
\hline \multirow[t]{2}{*}{ Dimensión } & \multirow{2}{*}{$\begin{array}{l}\text { Categorías } \\
\text { Indicador }\end{array}$} & \multicolumn{2}{|c|}{ Desfavorable } & \multicolumn{2}{|c|}{$\begin{array}{l}\text { Medianamente } \\
\text { favorable }\end{array}$} & \multicolumn{2}{|c|}{ Favorable } & \multicolumn{2}{|c|}{ TOTAL } \\
\hline & & Fcia & $\%$ & Fcia & $\%$ & Fcia & $\%$ & Fcia & $\%$ \\
\hline \multirow[t]{4}{*}{$\begin{array}{l}\text { Gestión } \\
\text { institucional }\end{array}$} & $\begin{array}{l}\text { 1. Tiene los recursos necesarios para } \\
\text { hacer su trabajo. }\end{array}$ & 4 & 26.7 & 9 & 60.0 & 2 & 13,3 & 15 & 100.0 \\
\hline & $\begin{array}{l}\text { 2. La información y la comunicación } \\
\text { fluyen oportuna y directamente. }\end{array}$ & 1 & 6.7 & 3 & 20.0 & 11 & 73.3 & 15 & 100.0 \\
\hline & $\begin{array}{l}\text { 3. Está orgulloso de pertenecer a esta } \\
\text { institución. }\end{array}$ & 4 & 26.7 & 8 & 53.3 & 3 & 20.0 & 15 & 100.0 \\
\hline & $\begin{array}{l}\text { 4. Ud. Es reconocido en proporción al } \\
\text { trabajo desempeñado. }\end{array}$ & 5 & 33.3 & 9 & 60.0 & 1 & 6.7 & 15 & 100.0 \\
\hline TOTAL & & 14 & 23.3 & 29 & 48.3 & 17 & 28.3 & 60 & 100.0 \\
\hline
\end{tabular}

Cuestionario CLIOUNing aplicado por el investigador al personal docente y administrativo.

El 48.3\% dela población indica quela interacción institucional es medianamente favorable, esto evidencia que el personal no está satisfecho con los recursos que le brinda la institución para que puedan realizar las labores propias de sus funciones, percibe una comunicación interna inadecuada el cual dificulta poder llevar a cabo sus actividades. Tiene un bajo sentido de identidad institucional, generando sentimientos de frustración ante orgullo y pertenencia. Considera que la institución no reconoce de manera proporcional los aciertos y equivocaciones del personal.

\section{Discusión}

EnladimensiónGestión Institucional,el 73.5\%de docentes muestran favorablemente en información y la comunicación que fluyen oportunamente y directamente, donde es concordante las ideas que hizo Esquivel, et al., (2020) que a raíz de acciones del hombre integrado en una organización, ante esta situación las relaciones son susceptibles de crear o suscitar escenarios de discrepancia, confrontación, ocasionada por diversos factores como mala comunicación, la ausencia del diálogo y escucha activa, liderazgo, entre muchos otros, en escenarios desfavorables se presentan resultados negativos en el clima organizacional, mientras el estudio realizado en la presente, el resultado es favorable, implica que las personas que laboran en dicha organización practican valores que inculcan para favorecer el clima organizacional.

Cuando Chiang, et al., (2017) al referirse que el Clima Organizacional es la descripción individual del marco social o contextual del cual forma parte una persona; son percepciones compartidas de políticas, prácticas y procedimientos organizaciones tanto formales como informales, permite afirmar sobre la buena gestión de recursos humanos depende de buenas acciones en las personas, por lo que Montoya et al., (2017) aclara que el recurso humano cumple un rol fundamental en el logro de metas de una institución.(Montoya et al., 2017).

En el caso de la dimensión Interacción Interinstitucional alcanzan una cifra cerca al 50\%, donde el $48.3 \%$ de docentes y administrativos demuestran medianamente satisfactorio en esta 
dimensión, estos resultados no son tan adecuados en una organización, por lo que es necesario lograr competencias como indica Cortés y Rosales (2019), por lo cual, las buenas relaciones personales son importantes en una institución, por eso, el desarrollo de las competencias socio afectivas favorece la adquisición de valores y conductas relacionados con el trabajo cooperativa, el logro de estas capacidades permitiría mayor acierto en las organizaciones, tal como indica Pupo et al., (2017), en consecuencia, en toda organización humana existe una relación bilateral positiva y estadísticamente significativa entre el clima organizacional y la satisfacción laboral, los resultados negativos pueden generar implicaciones psicológicas insatisfactorios en los trabajadores, como indica Chiang, Heredia, et al., (2017), por lo tanto, los resultados de esta investigación, permite que debe elevarse favorablemente el clima organizacional en la escuela, tal como recalca Zambrano et al., (2017) que el clima organizacional es muy importante en la gestión de las organizaciones es estudiado para obtener un alto desempeño laboral, eleva el compromiso y la cooperación entre los participantes del grupo de trabajo de manera satisfactorio, para tener resultados satisfactorios debemos aplicar el liderazgo en los directivos, tal como afirma Salguero y García (2017) el tipo del líder influirá en los trabajadores, interviniendo en el actuar de estos de tal forma que genera una motivación que permite el máximo desempeño en busca de la excelencia organizacional, lo mismo sostiene Castillo et al., (2019) si se tiene o se buscan altos niveles de liderazgo, podrían traducirse en mejoras del clima organizacional, por otra parte, Adrianzola et al., (2019), sostiene que el rol del líder incide positivamente sobre las conductas de apoyo y de orientación a las reglas y sobre la autonomía y profesionalismo, ambas dimensiones se potencien mutuamente, de forma tal que el clima mejore influyendo en la mejor disposición de los trabajadores entonces, las instituciones $\mathrm{u}$ organizaciones que poseen un clima organizacional agradable, debido a que permite a cada uno de los empleados desarrollar sus funciones de manera óptima (Cota, 2017), desde los resultados de la investigación, se comprende que el factor humano la más importante en una organización, a quien debemos prestar la debida atención para mantener elevado su nivel emocional y de satisfacción, impidiendo que llegue a realizar actividades que se vuelvan monótonas y sin sentido (Meza, 2017), mientras tanto más de la mitad de docentes se encuentra medianamente satisfechos dentro ámbito laboral, para ello, los directivos deberán mejorar el clima organizacional hasta encontrar resultados favorables como se encuentra en los estudios realizados en docentes, que ciertos resultados están satisfechos con el clima organizacional, indistintamente del sistema de valores (Riveros y Grimaldo, 2017), estos hallazgos permiten cada vez mejorar en las organizaciones su satisfacción con y en grupos de trabajo en las Instituciones Educativas.

Para tener éxito en nuestras organizaciones la motivación va acompañado en las personas, mientras el ser humano se sienta feliz y comprometido en su organización, se verá en el trabajador una satisfacción laboral y crecerá buenas relaciones interpersonales, por eso es fundamental el crecimiento del clima organizacional favorable (Pazmay y Ortíz, 2018; Diaz y Carrasco, 2018; Parra et al., 2018, Cardona et al., 2018). Los autores 
mencionados tienden a concluir la importancia que tiene el factor humano y su desempeño para mejorar el clima organizacional en el trabajo, lo propio la tesis que se plantea es propicio y oportuno que las entidades públicas y privadas incentiven a sus trabajadores mantener buenas relaciones y un adecuado clima favorable.

Como se muestra en los resultados de esta investigación, que en las tres dimensiones existe indicadores para ser mejoradas; en la dimensión gestión institucional un $11.6 \%$, en los retos personales un $11.1 \%$ y en la interacción un $23.3 \%$, estos datos son desfavorables, en consecuencia, necesita para revertir los resultados de la calidad de vida laboral de los trabajadores los cuales son factor clave para la productividad de una organización. Suárez, (2018), relaciona una participación activa de hombres y mujeres, Para González et al., (2018), tanto en los trabajadores del sector público. En Charry, (2018) y Rivera et al., (2018) mencionan así también quienes trabajan en el sector privado califican más alto este factor que los empleados del sector público en la identidad y la oportunidad de crecer en un clima organizacional favorable y aportar el crecimiento de la organización, Pedraza y Bernal, (2018), entonces es fundamental para el crecimiento del clima organizacional el liderazgo, el compromiso organizacional, el desempeño laboral, la motivación, el trabajo en equipo, la cultura organizacional, representan algunas de las líneas de investigación futuras en las organizaciones públicas y privadas (Pedraza, 2018).

Se afirma que toda organización requiere de una estrategia para mejorar el clima organización, entre ellos la actitud del trabajador, su comportamiento en la organización influyen directamente su mejora (Lapo y Bustamante, 2018), es así que en la dimensión de relaciones institucionales existe una carencia de interacción interinstitucional e interpersonales en los trabajadores, por lo que se puede expresar a mayores y adecuadas relaciones interpersonales entre compañeros y con los superiores representa ser el componente del clima organizacional que mayor impacto produce sobre la satisfacción del personal hacia su centro de trabajo (Soria et al., 2019) toda investigación contribuye grandemente hacia la mejora de las temáticas, el resultados de esta tesis serán tratados por otros investigadores para proponer su constante mejora y cultivar en los colaboradores generen confianza, empoderamiento y compromiso de los mismo para afrontar los retos presentes y futuros (Brito et al., 2020).

Sin duda, existen otras alternativas para aplicar en tiempos de COVID-19, tal como señala el autor que las Tecnologías de Información y Comunicación, resultan estratégicos para el desarrollo organizacional en la medida que contribuyen con el proceso de innovación y desarrollo tecnológico, dirigido hacia el mejoramiento de algunos aspectos clave de la organización que permiten incrementar su competitividad en los mercados globalizados (Panchi, 2018), sin embargo, los estudios acerca del tema deben ser profundizados, todo resultado favorable es percibida por el usuario, quienes califican su funcionalidad, califican la calidad de atención y ser satisfechos dentro de una organización humana, tal cual ocurre en los establecimientos de salud y educación dónde el cliente tiene la razón, lo propio debe evidenciar la dimensión técnica científica aguardando coherencia y correspondencia 
con la dimensión humana para lograr que se valore la calidad de la atención y eficiencia (Fernández et al., 2019; Ortiz et al., 2019), más así se puede afirmar en el campo educativo, así como las organizaciones de las entidades públicas y privadas, entonces, se concuerda con el planteamiento de Brito, (2018) y la constante mejora dependerá de la evaluación permanente del personal humano en las organizaciones cuando las personas trabajan en una organización y se sienten contentas con la función desempeñada, el compromiso con esta aumenta y mejoran los resultados (Chirinos et al., 2018), la educación y la formación humana será primordial para mejorar la cultura de mejora constante en las personas (López et al., 2019) para lo cual se pueden emplear estrategias de gestión de recursos humanos apropiadas y concretas para motivar y retener a la gente y mejorar su desempeño laboral (Luna et al., 2019), por eso la pedagogía es muy importante para un clima organizacional positivo (Niebles et al., 2019), por consiguiente las decisiones acertadas, contribuye en la mejora continua en el desarrollo humano (Hirsch, 2016) entonces toda organización deberá promover una acertada capacitación para promover un clima organizacional adecuado que sea sostenible con el tiempo (Bada et al., 2020).

\section{CONCLUSIONES}

Los resultados obtenidos en la investigación, se tiene que la mayoría de la población en estudio responden tener un clima medianamente satisfactorio, debiendo ser favorable para alcanzar mayores posibilidades para obtener un ambiente que favorezca en las mismas instituciones educativas, se muestran los resultados por dimensiones lo que permitió al investigador encontrar hallazgos altamente significativos en el campo del clima institucional.
En la dimensión gestión institucional los resultados presentados del promedio total, permiten apreciar mayor porcentaje $48.3 \%$ de satisfacción, donde se evidencia una percepción medianamente favorable.

En la dimensión retos personales los resultados presentaron mayor porcentaje de $68.8 \%$ de satisfacción donde se evidencia una percepción medianamente favorable del clima organizacional, todas ellas presentan una aceptación de mayor satisfacción.

En la dimensión interacción institucional los resultados presentan un promedio, que permiten apreciar un $48.3 \%$ de satisfacción, donde se evidenció una percepción medianamente favorable del clima organizacional.

En la percepción del clima organizacional en docentes y administrativos de la Institución Educativa Primaria de Salcedo Puno, la autoridad es aceptada y no impuesta; los resultados presentados del promedio total, nos permiten apreciar un mayor porcentaje de $53.9 \%$ de satisfacción, del total de la población analizada donde se evidencia una percepción medianamente favorable del clima organizacional; el segundo conglomerado representa el $30.3 \%$ del total de la población analizada el cual nos indica un clima favorable y el menor conglomerado, igual a $15.7 \%$ del total de la población investigada, representa la percepción del clima organizacional desfavorable, el lugar de decisión es amplio ya que puede tomarse en todos los niveles, decisiones acordes en los grados de responsabilidad; sin embargo, ello indica insatisfacción en cuanto a la necesidad de pertenencia, autonomía, poder y responsabilidad dentro de la dinámica organizacional. 


\section{REFERENCIAS}

Adrianzola, F. P., López, M. J., Rojas, R. A., y Bustamante, M. A. (2019). Percepción Del Clima Organizacional En Atención Perception of the Organizational Climate in Primary Attention of Health in the Region of the Maule, Chile. Revista Empresarial, 13(1):13-21. https:// doi.org/10.23878/empr.v13i01.132

Bada, O. F., Salas, R. M., Castillo, E. F., Arroyo, E. M., y Carbonel, C. E. (2020). Estrés laboral y clima organizacional en docentes peruanos. Medisur, 18(6), 1138-1144

Brito, C., Pitre, R., y Cardona, D. (2020). Clima Organizacional y su Influencia en el Desempeño del Personal en una Empresa de Servicio. Información Tecnológica, 31(1), 141-148. https://www.scielo.cl/pdf/infotec/v31n1/07180764-infotec-31-01-141.pdf

Brito, J. (2018). El clima organizacional como estrategia de mejora en una organización. RICEA Revista Iberoamericana de Contaduría, Economía y Administración, 7(13), 114-132. https://doi.org/10.23913/ricea.v7i13.114

Cardona,D.,Lamadrid,J.,yBrito,C.(2018).Lagestión y dirección del talento humano desde el análisis sobre clima organizacional y sus dimensiones. Un estudio de caso. Aglala, 9(1), 154-176. https://doi.org/10.22519/22157360.1185

Castillo, E. F., Medina, M. A., Bernardo, J. V., Reyes, C. E., y Ayala, C. I. (2019). Leadership and organizational climate in workers of health facilities of a micro-network in Peru. Revista Cubana de Salud Publica, 45(2), 1-13

Charry, H. O. (2018). La gestión de la comunicación interna y el clima organizacional en el sector público. Comunicación, 9(1)

Chiang, M. M., Fuentealba, I. I., y Nova, R. A. (2017). Relación entre clima organizacional $\mathrm{y}$ engagement, en dos fundaciones sociales, sin fines dde lucro, de la región del Bio Bio. Ciencia \& Trabajo, 19(59), 105-112. https:// scielo.conicyt.cl/pdf/cyt/v19n59/0718-2449cyt-19-59-00105.pdf

Chiang, M. M., Heredia, S. A., y Santamaría, E. J. (2017). Clima organizacional y salud psicológica del os trabajadores: Una dualidad organizacional
[Organizational climate and psychological health workers: An organizational duality]. Revista Dimensión Empresarial, 15(1), 73-86.

Chirinos, Y., Merino, V. H., y Martínez, C. (2018). El clima organizacional en el emprendimiento sostenible. Revista EAN, 84, 43-61. https://doi. org/DOI: https://doi.org/10.21158/01208160. n84.2018.1916 Disponible

Cortés, D. A., y Rosales, G. A. (2019). Propuesta pedagógica para el mejoramiento del clima organizacional. Encuentros, 17(01), 145-161. https://doi.org/10.21803/adnogsis.v6i6.196

Cota, J. A. (2017). Método para evaluar el Clima organizacional del área docente de un centro de Bachillerato Tecnológico de CD. Obregón, Sonora. Method to evaluate the organizational climate of the teaching area of a technological bachelor center of Obregon, Sonora. Ciencias Administrativas, 5(10), $010 . \quad$ https://doi. org/10.24215/23143738e010

Diaz, F. J., y Carrasco, M. E. (2018). Efectos del clima organizacional y los riesgos psicosociales sobre la felicidad en el trabajo. Contaduría y Administración, 63(4), 52

Esquivel, J., Martínez, G., y Silva, F. (2020). Clima organizacional. Aspectos básicos de su origen y definición. Ciencias de La Documentación, 6(2), 16

Fernández, D., Revilla, M. E., Kolevic, L. A., Cabrejos, I., Muchaypiña, I., Sayas, I., Chávez, L., y Mamani, V. A. (2019). Clima organizacional y satisfacción del usuario externo en los servicios de hospitalización del Instituto Nacional de Salud del Niño, 2017. Anales de La Facultad de Medicina, 80(2), 188-192. https://doi. org/10.15381/anales.802.15745

González, J. J., Rodríguez, M. T., y González, O. U. (2018). de la gran empresa Boyacense, estudio en el Valle de Sugamuxi, Colombia. Espacios, 39(37), 3. http://www.revistaespacios.com/ a18v39n37/a18v39n37p03.pdf

Hirsch, A. (2016). Comportamiento responsable en la investigación y conductas no éticas en universidades de México y España. Revista de La Educacion Superior, 45(179), 79-93. https:// doi.org/10.1016/j.resu.2016.06.005 
Lapo, M. C., y Bustamante, M. A. (2018). Incidencia del Clima Organizacional y de las Actitudes Laborales en el Comportamiento Prosocial de los Profesionales de la Salud del Guayas Ecuador. Información Tecnológica, 29(5), 245-258. http:// dx.doi.org/10.4067/S0718-07642018000500245

López, E., García, L. F., y Martínez, J. E. (2019). La gestión directiva como potenciadora de la mejora del clima organizacional y la convivencia en las instituciones de educación media superior. RIDE Revista Iberoamericana Para La Investigación y El Desarrollo Educativo, 9(18), 792-812. https:// doi.org/10.23913/ride.v9i18.471

Luna, O. Y., Armendariz, C. R., y Andrade, F. (2019). Cultura y clima organizacional en el desempeño laboral de empleados de los distritos costeños de educación en el Ecuador. Universidad $y$ Sociedad, 5, 327-335. http://rus.ucf.edu.cu/ index.php/rus\%0ARESUMEN

Meza, E. (2017). Análisis en la percepción del género entre clima organizacional y satisfacción laboral del sector industrial - México.Comuni@ cción - Revista de Investigación En Comunicación y Desarrollo, 8(2), 148-158-158

Montoya, P., Bello, N., Bermudez, N., Burgos, F., Fuentealba, M., y Padilla, A. (2017). Satisfaccion laboral y su relacion con el clima organizacional en funcionarios de una Universidad Estatal Chilena. Ciencia \& trabajo [revista en Internet] 2017 [acceso 2 enero de 2018]; 19(58): [7-13]. Ciencia y Trabajo, 19(58), 7-13. http://www.scielo.cl/scielo.php? script $=$ sci_ arttext\&pid=S0718-24492017000100007\&lng= en\&nrm=iso\&tlng=en

Niebles, W. A., Hoyos, L. D. C., y De-La-Ossa, S. J. (2019). Clima Organizacional y Desempeño Docente en Universidades Privadas de Barranquilla. Saber, Ciencia y Libertad, 14(2), 283-294. https://doi.org/10.18041/2382-3240/ saber.2019v14n2.5893

Ortiz, L., Ortiz, L. E., Coronell, R. D., Hamburger, K., y Orozco, E. (2019). Incidence of organizational climate on labor productivity in institutions providing health services (IPS): A correlational study. Revista Latinoamericana de Hipertension, 14(2), 187-193
Panchi, V. P. (2018). La incidencia del clima organizacional en el desarrollo de las organizaciones postmodernas. Sapienza Organizacional, 5(9), 173-188.i

Parra, M., Duran, S. E., Márceles, V., Yarzagaray, J., Valenzuela, I., Payares, K., Alvarino, C., Navarro, N., Charris, P., y Ramirez, J. (2018). Factores del clima organizacional existentes en el área administrativa en empresas de Barranquilla. Espacios, 39(50), 21. http://www.revistaespacios. com/a18v39n50/18395021.html

Pazmay, S. G., y Ortíz, Á. R. (2018). Clima Organizacional en las Industrias ecuatorianas de calzado. Cuadernos Latinoamericanos de Administración, 14(26), 39-50. https://doi. org/10.18270/cuaderlam.v14i26.2606

Pedraza, N. A. (2018). El clima organizacional y su relación con la satisfacción laboral desde la percepción del capital humano. Revista Lasallista de Investigación, 15(1), 90-101. https://doi. org/10.22507/rli.v15n1a9

Pedraza, N. A., y Bernal, I. (2018). El clima organizacional en el sector público y empresarial desde la percepción de su capital humano. Espacios, 39(13), 16. http://www.revistaespacios. com/a18v39n13/a18v39n13p16.pdf

Pupo, B., Velásquez, R., y Tamayo, M. ángel. (2017). Relación entre clima organizacional y satisfacción laboral en empresas holguineras. Ciencias Holguín, 23(4), 71-83

Rivera, D. A., Rincón, J. E., y Flores, S. R. (2018). Percepción del Clima organizacional: Un análisis desde los Macroprocesos de una ESE. Revista Espacios, 39(19).

Riveros, P. N., y Grimaldo, M. P. (2017). Valores y clima organizacional en docentes de un instituto de educación superior de Lima. Ciencias Psicológicas, 11(2), 179. https://doi. org/10.22235/cp.v11i2.1489

Sagredo, E., y Castelló, A. (2019). Gestión directiva y clima organizacional en la educación de personas adultas en Chile. Actualidades Investigativas En Educación, 19(2), 1-23. https:// doi.org/10.15517/aie.v19i2.36895 
Salguero, N. G., y García, C. P. (2017). Influencia del liderazgo sobre el clima organizacional en las instituciones de educación superior. Boletín Redipe,6(4),135-149.https://dialnet.unirioja.es/ servlet/articulo? codigo $=6119351 \% 0$ Ahttps: $/ /$ dialnet.unirioja.es/descarga/articulo/6119351. pdf

Soria, A. C., Pedraza, N. A., \& Bernal, I. (2019). El clima organizacional y su asociación con la satisfacción laboral en una institución de educación superior. Acta Universitaria, 29, 1-14

Suárez, S. (2018). Clima organizacional entre los trabajadores del Hospital General "La Villa": hospital de segundo nivel de atención de la
Ciudad de México Organizational climate among the workers of the "Hospital General La Villa": Hospital of the second level of care in. Rev Cubana Salud Pública, 44(4), 97-111. http://scielo.sld.cuhttp//scielo.sld.cu

Zambrano, J., Ramón, M. A., y Espinoza, E. (2017). Estudio Sobre El Clima Organizacional En Docentes De La Universidad Técnica De Machala. Universidad y Sociedad, 9(2), 163-172 